\title{
DeVeloping A Synthetic COMPOSITE MEMBRANE For CleFt PALATE RePAIR
}

Faiza Sharif*1, Sabiniano Roman ${ }^{2}$, Anila Asif ${ }^{1}$, Giulia Gigliobianco ${ }^{2}$, Sarah Ghafoor ${ }^{3}$, Muhammad Tariq ${ }^{4}$, Saadat Anwer Siddiqui ${ }^{1}$, Farrukh Mahmood ${ }^{5}$, Nawshad Muhammad ${ }^{1}$, Ihtesham Ur Rehman ${ }^{1,2}$, Sheila MacNeil ${ }^{2}$

1-Interdisciplinary Research Centre in Biomedical Materials, COMSATS University Islamabad, Lahore Campus, Pakistan. 2- Department of Materials Science \&Engineering, Kroto Research Institute, University of Sheffield, Broad Lane, Sheffield, UK. 3- Department of Oral Biology, University of Health Sciences Lahore, 4- Department of Biology, Syed Babar Ali School of Science and Engineering, Lahore University of Management Sciences, Lahore. 5- Department of Pediatric Plastic Surgery, Children Hospital, Lahore.

*Corresponding Author: Dr Faiza Sharif: Interdisciplinary Research Centre in Biomedical Materials, COMSATS University Islamabad, Lahore Campus, Defence Road, off Raiwind Road, Lahore, Pakistan. E mail: faizasharif@cuilahore.edu.pk

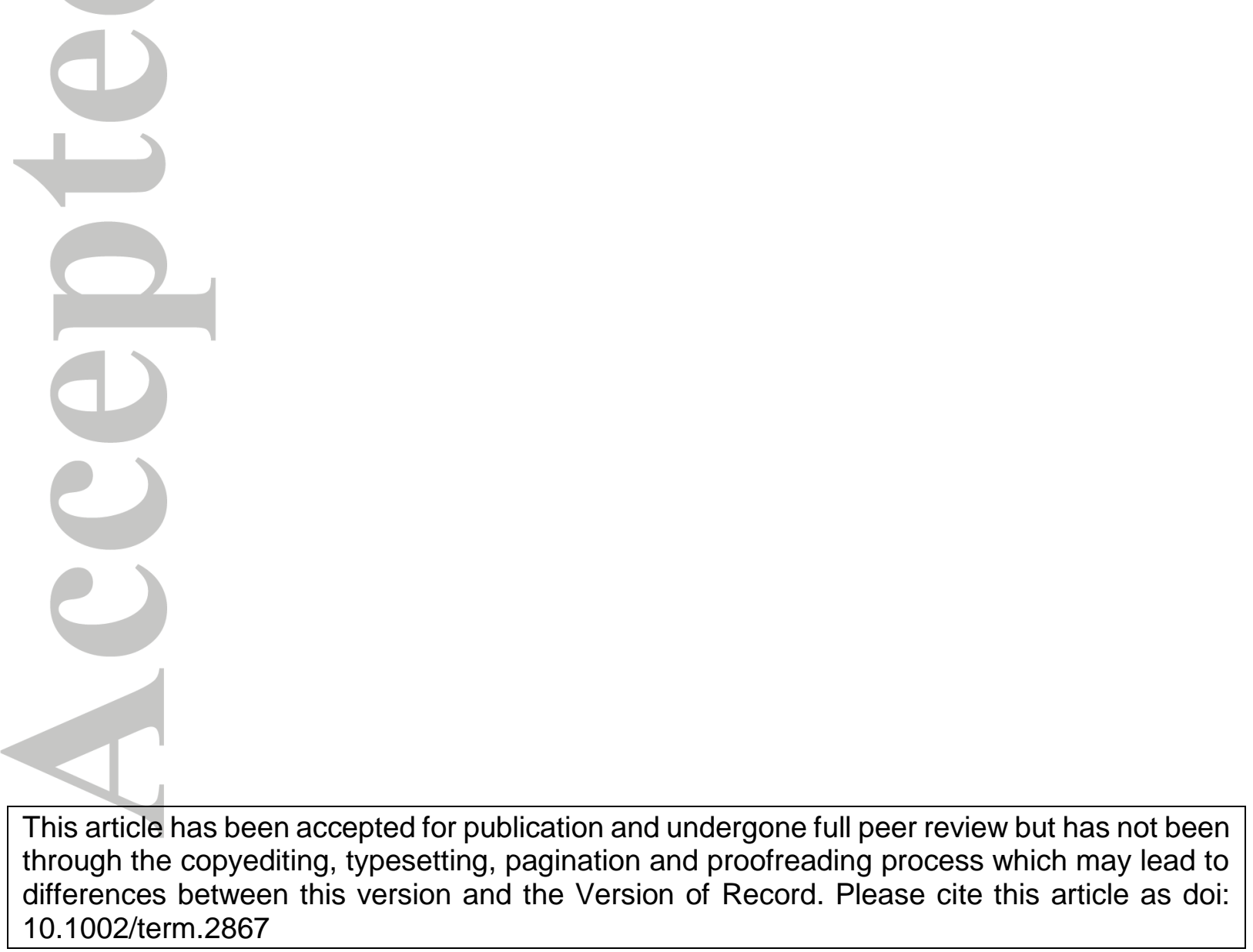




\section{Abstract}

An oronasal fistula is a passage between the oral and nasal cavity. Currently, surgical procedures use mucosal flaps or collagen grafts to make a barrier between oral and nasal cavities. Our aim was to develop a cell-free synthetic repair material for closure of nasal fistulas. We surface functionalized electrospun polyurethane (PU) and poly-L-lactic acid (PLLA) and composite polymer (PU-PLLA) membranes with acrylic acid through plasma polymerization. Membranes were treated in a layer-by-layer approach to develop highly charged electrostatic layer which could bind heparin as a pro-angiogenic glycosaminoglycan. The properties were evaluated through physical, chemical and mechanical characterization techniques. Cytotoxicity was tested with MC3T3 pre-osteoblast cell lines for 3, 7 and 14 days and vasculogenesis was assessed by implantation into the chorio-allantoic membrane in chick embryos for 7 days. In vivo biocompatibility was assessed by subcutaneous implantation in

rats for 1, 3 and 6 weeks. The membranes consisted of random fibres of PLLA-PU with fibre diameters of $0.47 \mu \mathrm{m}$ and $0.12 \mu \mathrm{m}$ respectively. Significantly higher cell proliferation and migration of MC3T3 cells at 3, 7 and 14 days were shown on plasma coated membranes compared to uncoated membranes. Further, it was found that plasma coated membranes were more angiogenic than controls. In vivo implantation of membranes in rats did not reveal any gross toxicity to the materials and wound healing was comparable to the native tissue repair (sham group). We therefore present a plasma functionalized electrospun composite polymer membrane for use in the treatment of fistulas. These membranes are flexible, non-cytotoxic and angiogenic and we hope should lead to permanent closure of oronasal fistula.

Key Words: Polymer composite, electrospinning, plasma polymerized membranes, fistula repair, cleft palate repair 


\section{INTRODUCTION}

Congenital deformities of the lip and palate are very common with a prevalence of around 1 in 700 live births in the UK and USA and 1 in 500-700 worldwide (Mossey, 2004). Surgical intervention is almost always necessary to repair the soft and hard palate defects. Successful repair seeks to achieve complete separation of the oral and nasal cavities and this helps with acquisition of speech. However even after the most careful surgical procedures a persistent gap or hole sometimes appears between the two cavities termed a palatal fistula (Sadhu, 2009). Occasionally urgent repairs are needed with large sized fistula as these can lead to nasal emissions and leakage of fluid and food into the nasal cavity. Fistulae are significantly higher in patients who have undergone repair of bilateral clefts of the lip and palate than for patients who have undergone repair of a unilateral cleft lip and palate (Hosseinabad et al., 2015). Additionally, palatal fistulae are more common in cases of combined cleft lip-cleft palate, compared to cleft palate alone (Hardwicke, Landini, \& Richard, 2014). The most common location for a fistula is at the soft palate-hard palate junction (Bykowski, Naran, Winger, \& Losee, 2015).

There are a range of surgical techniques which have been developed to treat fistulae. Their use will depend on the size of the fistula and its location and the cause of the defect. Depending on the type of surgical procedure performed the rate of fistula occurrence has been reported to be $4.9 \%$ (Bykowski et al., 2015) or up to $17.7 \%$ (Kahraman et al., 2014). These techniques range from the use of a pedicle buccal fat pad (BFP) (de Castro, de Souza, \& Fernandes Santos, 2015); conchal cartilage graft to repair large fistula [8]; multilayered repair of the whole palate (Funayama et al., 2014); or a tri-layered repair where the bone and cartilage are harvested from the nasal septum of the same patient and are used to create the middle layer sandwiched between two vascular layers (Hudson \& Pickett, 2015).

Acellular collagen grafts have been used in the 3-layer closure described above during a primary palatoplasty surgery. These are viewed as an effective and risk free strategy in preventing oronasal fistulation (Hudson \& Pickett, 2015). Acellular dermal matrix allografts are also considered safe and effective for use in closure of large (more than $15 \mathrm{~mm}$ ) and recurrent oronasal fistulae in the hard palate (Badami, Kreke, Thompson, Riffle, \& Goldstein, 2006). However, cadaveric decellularised dermis is not universally available and as yet there are no well-established tissue banks in Pakistan. Alloderm commercial donor dermis is available as an import item but extremely expensive and a patch of $7 \times 5 \mathrm{~cm}$ is worth 100, 000 PK rupees (equivalent to 100 US dollars).

Accordingly, our aim was to develop a synthetic biomaterial which could be used as an alternative to cadaveric skin or collagen grafts or patient's tissues to assist in the closure of nasal fistulas. There are a range of polymers routinely used for tissue guides because they are inherently biocompatible and one can choose their physiochemical properties and tailor them to the application required (Porter, Ruckh, $\&$ Popat, 2009). Such polymers include Poly(-hydroxyl acid) related polymers, such as poly-L-lactic acid (PLLA) (Badami et al., 2006), poly glycolic acid (PGA) (Wang et al., 2012), poly-caprolactone (PCL) (Yoshimoto, Shin, Terai, \& Vacanti, 2003) and their copolymers which have been extensively 
studied for use in scaffold fabrication (Coombes \& Meikle, 1994). Porous PCL-HA scaffolds (Chuenjitkuntaworn et al., 2010), poly (ع-caprolactone) coated in polydopamine (Zhang, Giese, Prukop, \& Grunlan, 2011), poly (hydroxybutyrate) (PHB) and poly (hydroxybutyrate-co-hydroxyvalerate) (PHBV) (Sombatmankhong, Sanchavanakit, Pavasant, \& Supaphol, 2007) are a few examples of such polymers.

Additionally, polyurethanes (PUs) are very attractive because of their flexibility. They belong to a family of materials with a diversity of chemical compositions, mechanical properties, tissue-specific biocompatibility, and biodegradability (Zdrahala \& Zdrahala, 1999). Their segmented block copolymer character endows them with a wide range of versatility in terms of tailoring their physical properties, blood and tissue compatibility making them attractive for both hard and soft tissue engineering (Alperin, Zandstra, \& Woodhouse, 2005; Macocinschi, Filip, \& Vlad, 2011). Their ability to distend and recoil without wear makes them attractive for pediatric applications like cleft palate repair and alveolar ridge augmentation, where materials with high tensile strength are required.

Accordingly, we explored the potential of a PU electrospun membrane combined with PLLA to produce an elastic polymer composite to encourage cellular infiltration and matrix deposition and good in vivo tissue integration. Further we also explored a surface coating approach to bind heparin to the material to encourage rapid ingrowth of blood vessels and accelerate wound healing.

\section{Materials and methods}

\subsection{MEMBRANE FABRICATION BY ELECTROSPINNING}

The cospun scaffolds were made by spinning the PU and PLLA from different syringes at the same time depositing both fibers on the rotating electrospun collector giving rise to intermingled membranes. PU Z3 (Biomer Technologies, Cheshire, UK) was dissolved in 70 / 30\% dimethylformide: tetrahydrofuran (DMF: THF), respectively, in a concentration of 15\% w/v. PLLA (Goodfellow, Cambridge, UK) was dissolved in dichloromethane (DCM) (Sigma-Aldrich, UK) in a 10\% w/v ratio. The solutions were prepared at room temperature on a mechanical stirrer overnight. The following day, $32 \mathrm{~mL}$ of PU solution was electrospun with a $50 \mathrm{~mL}$ syringe fitted with a multichannel needle holder, using 7 stainless steel blunt needles. It was placed horizontally on a programmable syringe pump (Kent Scientific, USA) with a discharge rate of $280 \mathrm{ul} / \mathrm{min}$ at $17 \mathrm{kV}$. For cospinning, 5ml PLLA was loaded in one $5 \mathrm{ml}$ syringe on the right-hand side with a discharge rate of $40 \mathrm{ul} / \mathrm{min}$. The syringe was supplied with $17 \mathrm{kV}$ by a high voltage power supply (Genvolt, UK). The fibrous membrane was collected on a rotating drum $16 \mathrm{~cm} \times 10 \mathrm{~cm}$ in diameter at a distance of $17 \mathrm{~cm}$ from the needle tips of both polymers. The rotation speed of drum was $300 \mathrm{rpm}$. An $18 \mathrm{~cm} \mathrm{X} 16 \mathrm{~cm}$ mat of approximately $150 \mu \mathrm{m}$ thickness was formed. Membranes were produced at a room temperature of around $21.5^{\circ} \mathrm{C}$. Single polymer mats of PU Z3 and PLLA were produce, as previously described [26, 28], for comparison of physical, chemical and mechanical properties. 


\subsection{A LAYER BY LAYER TREATMENT TO HEPARIN COAT THE MEMBRANES}

The electrospun PU, PLLA and cospun PU-PLLA membranes were initially plasma polymerized with polyacrylic acid and then treated in a layer by layer approach, finally being loaded with heparin ((SigmaAldrich, USA) to enhance the ability of the membranes to promote angiogenesis. A layer-by-layer coating was achieved by building up layers of acrylic acid then polyethylenimine (PEI). The method of polymerization was as reported previously (Gigliobianco, Chong, \& MacNeil, 2015). Briefly, first 98\% polyacrylamide (Sigma-Aldrich, USA) in deionized water was used then the PU- PLLA mat was inserted into the glass chamber of the plasma rig. The initial pressure was adjusted at 3.0_10-2 mbar. The vacuum valve was then closed for $30 \mathrm{~s}$ and the final pressure was set at $4.2 \_10^{-1} \mathrm{mbar}$. The power was then switched on for $20 \mathrm{~min}$ by an RF-plasma at $13.56 \mathrm{MHz}$ delivering at $15 \mathrm{~W}$. Plasma polymerized electrospun membranes were transferred into an aqueous solution of $0.5 \% \mathrm{w} / \mathrm{v}$ PEI (Sigma-Aldrich, UK) at pH 9 for 30 min at room temperature. Membranes were washed four times with de- ionized water and they were immersed in a $100 \mathrm{U} / \mathrm{mL}$ aqueous solution of Heparin for $30 \mathrm{~min}$ at room temperature. Membranes were washed again four times with de ionized water and these steps were repeated each time to produce seven layers, with the last layer being PEI. After the layer-by-layer coating, membranes were dipped into $2 \mathrm{~mL}$ of $100 \mathrm{U} / \mathrm{mL}$ heparin aqueous solution for $4 \mathrm{~h}$ at room temperature. Afterwards, membranes were placed in fresh phosphate buffer saline (PBS) solution for $24 \mathrm{~h}$.

\subsection{SCANNING ELECTRON MICROSCOPY (SEM)}

After functionalization with PEI and heparin, PU, PLLA and PU-PLLA membranes were dried at room temperature. Samples were then gold coated using a sputter coater from Quorum Technologies. Samples were imaged using TESCAN Vega3 LMU scanning electron microscope (SEM) using ETD detector and SE mode. SEM images were acquired using an acceleration voltage of $15 \mathrm{kV}$ with a beam intensity of $4 \mathrm{pA}$.

\subsection{Fiber thickness AND PORE Size ANALYSis OF PU-PLLA UnCOATED AND COATED MEMBRANE}

Fiber thickness was measured using Image-J software. Images at a magnification of 1000X were used for fiber thickness diameter. In the case of composite membranes, identification of each polymer was done based on the thickness and appearance of fibers (Gigliobianco et al., 2015), and 30 fibers from each polymer were measured. Separate membranes made of either PU or PLLA after being plasma coated or uncoated were analysed for fiber thickness and pore size and compared with cospun plasma coated and uncoated membranes of PU-PLLA.

This article is protected by copyright. All rights reserved. 


\subsection{FOURIER TRANSFORM INFRARED SPECTROSCOPY ANALYSIS (FTIR)}

PU, PLLA and PU-PLLA membranes were characterized by Fourier transform infrared spectroscopy analysis (FTIR) (FTIR, Nicolet 6700, USA) by using photoacoustic mode. All spectra were collected with wave number ranging from 4,000 to $400 \mathrm{~cm}^{-1}$ at resolution of $8 \mathrm{~cm}^{-1}$ averaging 256 scans.

\subsection{ASSESSMENT OF HYDROPHOBICITY/HYDROPHILICITY OF MEMBRANES}

To measure the difference in the hydrophobicity or hydrophilicity of the membranes droplet image analysis was performed by directly measuring the contact angle (CA) from images of the droplet. This analysis was performed using a goniometer, which is usually composed of three parts: a background light, a sample stage and a camera. A liquid droplet was carefully placed onto the surface of PUPLLA uncoated and coated membranes, an image of the droplet was captured and analyzed to determine a CA value. In this process, the background light, sample stage and camera were aligned to ensure accurate CA measurement.

\subsection{Mechanical Properties of Membranes}

Characterization of the biomechanical properties of the fabricated membranes was investigated by uniaxial tensile testing using a tensiometer (BOSE Electroforce test instruments, Minnesota, USA). To study the elastic properties, electrospun PU, PLLA and cospun PU-PLLA membranes were distended and relaxed for five cycles as shown in Figure 3 and then the ultimate tensile strength was assessed. Suture retention was also undertaken. Samples were cut keeping uniform width and length which were measured for each sample together with thickness, and then were clamped between two grips of tensiometer. The length between the clamps was kept uniform for all samples. A load cell of $45 \mathrm{~N}$ was used.

For the tensile test, a ramp test was applied as previously mentioned [26] at a rate of $0.1 \mathrm{~mm} / \mathrm{s}$ and a displacement of $7 \mathrm{~mm}$ (stress and strain were normalized by area and length of the sample respectively). Ultimate tensile strength was measured as the plateau at the first failure point. For the cyclic test, the material was stretched at a rate of $1 \mathrm{~mm} / \mathrm{s}$ and displacement was adjusted to $25 \%$ of its original length. Measurements were repeated at 5 cycles for each sample. Plastic deformation was measured as the initial \% of strain for each samples at the second cycle without an increase on stress due to this plastic deformation. Suture retention tests were also performed for each of the samples by taking a sample size of $1 \times 0.5 \mathrm{~cm}$. A non degradable suture was passed through the samples at a point approximately 0.25 $\mathrm{cm}$ from the top edge at the mid point of the width. Each membrane was clamped so that the gap between the two grids was $0.75 \mathrm{~cm}$. The data was obtained by plotting the max strength achieved before the suture ripped through the membrane as N. Porcine skin tissue was used as control for all tests.

\subsection{ASSESSMent of Cell Compatibility}

Cell culture on composite membranes was conducted using the mouse pre-osteoblast cell line (MC3T3 ATCC ${ }^{\circledR}$ CRL-2593 ${ }^{\mathrm{TM}}$ ). MC3T3 were expanded in Alpha minimum essential medium ( $\left.\alpha \mathrm{MEM}\right)$ (Thermo Fisher Scientific, USA) supplemented with 10\% fetal bovine serum (FBS) (Sigma Aldrich 
USA), $100 \mathrm{ug} / \mathrm{mL}$ of penicillin and streptomycin (Sigma Aldrich, Life Sciences USA). MC3T3s were expanded in culture flasks (Corning Biosystems). Cells were grown in a humidified incubator at $37^{\circ} \mathrm{C}$ with $5 \% \mathrm{CO}_{2}$ with fresh media changes performed every 2-3 days. Cells were grown to $90 \%$ confluency and then detached using trypsin-EDTA (Sigma Aldrich, USA). MC3T3 cells were used between passages 4-7. To analyze the viability and proliferation of osteoblastic cells on PU-PLLA plama coated and non coated membranes, cells were seeded at a density of 50,000 cells per sample in 24 well plates, separate replicate plates were used for each time point. Prior to cell seeding, the membranes were sterilized with ethanol for $1 \mathrm{~h}$, washed twice with PBS for $15 \mathrm{~min}$. Cells cultured without membranes were used as controls.

\subsubsection{ALAMAR BLUE ASSAY}

In order to quantify cell viability and proliferation, fluorescent measurements of Alamar Blue were obtained after 3, 7 and 14 days. For each time point, cell seeded PU-PLLA membranes were carefully washed with PBS and $0.5 \mathrm{ml}$ of $60 \mathrm{ug} / \mathrm{ml}$ Alamar Blue solution (Sigma Aldrich, UK) (diluted with $\alpha$ MEM-FBS ) was added followed by incubation at $37^{\circ} \mathrm{C}$ for $3-4 \mathrm{~h}$ absorbance was measured at $570 \mathrm{~nm}$ using a fluorescence plate reader (Bio-TEK, NorthStar Scientific Ltd, UK). Alamar Blue is an oxidation-reduction (REDOX) indicator that both fluoresces and changes color in response to cellular metabolic activity. Growth related reduction of the substrate causes the REDOX indicator to change from an oxidized (blue) form to a reduced (red) form. After the measurements were taken, samples were fixed for other studies.

\subsubsection{HISTOLOGY}

Histological samples were prepared from the cross section of unseeded and MC3T3-seeded membranes. Samples were fixed with $3.7 \%$ formaldehyde and washed 3 X PBS for $15 \mathrm{~min}$ each. They were then embedded in paraffin wax and $5 \mu \mathrm{m}$ sections were obtained with a Leica Microtome, and stained for Hematoxylin and Eosin (H\&E). Basically, the membranes were dehydrated in an ascending series of ethanol concentrations and then cleared in xylene for 3-4 min only, then the slides were stained with hematoxylin washed under running water and dehydrated with 50\% alcohol and counter stained in eosin in 50\% ethanol and then further dehydrated with absolute alcohol, cleared in xylene for $1 \mathrm{~min}$. Slides were then covered with DPX (Sigma-Aldrich, USA) and a glass coverslip to be imaged with a light microscope (Motic, China). Samples were observed with a Leica optical microscope using Kohler illumination at 20X objective magnification and scaled at $100 \mu \mathrm{m}$.

\subsection{ASSESSMENT OF ANGIOGENIC PROPERTIES OF COATED MEMBRANES USING THE CHORIO- ALLANTOIC MEMBRANE (CAM) ASSAY.}

Fertilized chicken (Gallus domesticus) eggs were purchased from VRI Lahore and incubated from day 2 of fertilisation until day 8 at $37^{\circ} \mathrm{C}$ in a humidified egg incubator (HHD 435, China). At day 8 , a square window $\left(1 \mathrm{~cm}^{2}\right)$ was cut into the shell and lifted, and a $1 \mathrm{~cm}^{2}$ surface modified electrospun membrane 
was placed onto the CAM. Half of the samples were fully functionalised prior to implantation, and then washed. The other samples were uncoated PU-PLLA membranes. Each egg was implanted with one membrane only. The shell window was replaced with parafilm (Bemis Flexible Packaging, USA) and sealed with adhesive tape. After implantation, eggs were placed again at $37^{\circ} \mathrm{C}$ in a $40 \%$ humidified incubator until day 14. At day 14, membranes were retrieved and the eggs were sacrificed.

Angiogenesis was quantified by taking light microscope pictures just before membrane retrieval and blindly scored by four independent assessors using histological images of the retrieved membranes.

\subsubsection{Histology OF CAM}

The membranes harvested from the CAM after 7 days of implantation were processed for histology. Membranes were fixed in 3.7\% paraformaldehyde (Sigma Aldrich, USA) in PBS overnight. Next, they were processed either for cryo-sectioning or for paraffin embedding. The samples were placed into molds for cryo-sectioning filled with OCT solution (Leica, Germany). They were left to freeze at $80^{\circ} \mathrm{C}$ and 20 um sections were cut with a cryostat (Leica CM1860UV (Leica Germany)). Slides were then stained trichrome stain, according to the standard protocol for frozen slides (Ott et al., 2008). Quantification of cell infiltration and angiogenesis of each membrane after the CAM assay was based on the trichrome histology. For assessment of cell infiltration and angiogenesis, three independent assessors scored 20 images for cell infiltration and 12 images for angiogenesis. The assessors did not know the nature of the samples they viewed. The histology images were scored from 1 (no cell infiltration) to 3 (massive cell infiltration). Angiogenesis was assessed by counting the number of blood vessels per slide.

\subsubsection{SEM IMAGES OF IMPLANTS HARVESTED FROM CAM}

Harvested implants were fixed in $3.7 \%$ glutaraldehyde and dehydrated in a graded series of ethanol then transferred into t-butyl alcohol and dried. Samples were coated with gold and were examined under a scanning electron microscope.

\subsection{IN VIVO IMPLANTATION OF MEMBRANES}

The animal experiments were approved by the Animal Care and Use Committee of the University of Health Sciences, Lahore, Pakistan. Two samples were implanted in each male Wistar rat on each side of the back. 2 groups of samples were implanted - surface functionalized PU-PLLA membranes and plain PU-PLLA membranes and also one group of negative controls. Negative controls were sham operated rats and were not implanted with anything, only a surgical wound pocket was made and then closed by applying sutures just as in treatment groups. The implants were kept in place for either 1, 3 or 6 weeks and 12 rats were used for each of the 3 groups (36 rats in total). Animals were placed in anesthetic; $100 \%$ isoflurane (Isoba) and kept under isoflurane anesthesia via a nose cone. A small area of skin at the back of the animal was shaved and disinfected, the skin was incised and flaps of the subcutaneous layer were raised (Figure 6aA-C). Implants were securely placed inside the created 
pocket-like space with the help of fine forceps then subcutaneous layer and skin were closed with nonresorbable sutures. Animals were weaned from anesthesia and observed for recovery (Figure 6aD-E).

\subsubsection{ANIMAL SACRIFICE, SAMPLE FIXATION AND HISTOLOGY}

At weeks 1, 3 and 6 after implantation, 4 animals from each group were sacrificed using an overdose of anesthesia. Dorsal skin pieces of $2 \mathrm{~cm}^{2}$ containing implants at the underside were explanted (Figure 6a F-G). Samples were fixed in 3.7\% paraformaldehyde and paraffin embedded for histology. Sections of $6 \mu \mathrm{m}$ thick were cut from the paraffin embedded samples with a microtome (Leica TP 1020 Automatic Tissue Processor) and placed on Superfrost ${ }^{\circledR}$ plus slides (Menzel-Gläser, Denmark). Sections were processed for H\&E staining, as above, for imaging.

\subsection{STATISTICAL ANALYSIS}

Unless stated otherwise, all experiments were conducted at least three times in triplicate. All data presented refer to standard error of the mean \pm (sem). In order to check for any statistically significant differences, a one-way ANOVA or students $t$ test was performed, multiple comparison post hoc test

was done where needed. Results with $p$-values of $<0.001$ were considered statistically significant. All data were analyzed using Graphpad Prism 7.0 software.

\section{Results}

\subsection{MORPHOLOGICAL CHARACTERIZATION}

SEM of PU-PLLA co-spun membranes showed randomly oriented fibers with a mixture of thin and thick fibers (Figure 1A). Fiber thickness analysis revealed that there were two distinct populations of fibers some thicker and other thinner ones when measured through image $\mathbf{J}$ software (Figure 1A). Fibre thickness was analysed from electrospun PU fibers and PLLA membranes separately and compared to cospun PU-PLLA fibres. PU fibres were thin with an average thickness of $0.2 \mu \mathrm{m}$ and PLLA fibres were thicker with an average thickness of $0.5 \mu \mathrm{m}$. Students' T test revealed that the difference between the means of PU and PLLA fibers was significant with $95 \% \mathrm{CI}$ of diff. with a p value of $<0.0001$.

Plasma coating affected the thickness of PU-PLLA cospun fibers and differences between the thickness of fibers between PU-PLLA cospun uncoated and coated were significant (Figure 1A). The mean thickness of uncoated PLLA fibers was $0.112 \mu \mathrm{m}$ and coated PLLA fibers was $0.169 \mu \mathrm{m}$ whereas the mean thickness of uncoated PU fibers was $0.417 \mu \mathrm{m}$ and coated PU fibers was $0.474 \mu \mathrm{m}$ Figure 1B. One way ANOVA analyses of the samples suggested that the differences between uncoated and plasma coated samples were significant with a $\mathrm{P}$ value of $<0.0001$. Multiple comparisons post hoc test also suggested significant differences between the means of PU uncoated vs PU coated fibers and PLLA uncoated vs PLLA coated fibers. Pore size was measured to be $5.715 \pm 0.4002 \mu \mathrm{m}$ in uncoated and 4.09 $\pm 0.3043 \mu \mathrm{m}$ in plasma coated samples. The pore size was significantly higher in uncoated membranes (results not shown). 
Surface morphology analysis to evaluate hydrophobicity / hydrophilicity after before and after plasma coating included CA measurement which describes the behavior of a liquid droplet on a solid surface in air, and is defined as the angle between the tangent at the three phase point and the solid surface. The left and right contact angle of water droplet on the surface of PU-PLLA uncoated membrane was measured as $99.6^{\circ}$ and $97.5^{\circ}$ respectively (Figure 1B) and that of PU-PLLA coated membrane was $63.1^{\circ}$ and $58.7^{\circ}$ respectively (Figure $\left.1 \mathrm{~B}\right)$.

\subsection{Chemical Characterization}

FTIR was used to identify the chemical structural characterization of the cospun PU-PLLA membranes. The representative FTIR spectra of PU and of PLLA are shown in Figure 2A, and the combined spectra of PU-PLLA (uncoated and coated) membranes are presented in Figure 2B.

For PU (Z3), as shown in Figure $2 \mathrm{~A}$ and B, the peak at $3325 \mathrm{~cm}^{-1}$, which indicates the N-H group in urethane (-NHCOO-), is attributed to the stretching $\mathrm{v}(\mathrm{N}-\mathrm{H})$. The peak at $3124 \mathrm{~cm}^{-1}$ was the overtone of $1532 \mathrm{~cm}^{-1}$ and attributed to the $\mathrm{v}(\mathrm{C}-\mathrm{H})$ in benzene ring of aromatic PU.

The peaks at 2932 and $2856 \mathrm{~cm}^{-1}$ belong to the asymmetric and symmetric vibration of $-\mathrm{CH}_{2}$ of the polyether of Z3 and PLLA. The carbonyl absorption region was observed in between 1760 and 1704 $\mathrm{cm}^{-1}$, the carbonyl absorption band classified into two peaks. The peak due to bonded $\mathrm{C}=\mathrm{O}$ stretching was at $1704 \mathrm{~cm}^{-1}$ and the characteristic absorption bands at $1760 \mathrm{~cm}^{-1}$ and $2920 \mathrm{~cm}^{-1}$ are attributed to the stretching vibrations of the $\mathrm{C}=\mathrm{O}$ and $\mathrm{CH}$ group of PLLA, respectively.

The peak at $\sim 1194 \mathrm{~cm}^{-1}$, attributed to C-O-C asymmetric vibrations linked with asymmetric $\mathrm{CH} 3$ rocking vibrations, in the region (1300-1000 $\mathrm{cm}^{-1}$ ), which is mainly related to the C-O-C stretching in PLLA.

The peak at $1595 \mathrm{~cm}^{-1}$ was assigned to $\mathrm{v}(\mathrm{C}=\mathrm{C})$ in the benzene ring and the $1419 \mathrm{~cm}^{-1}$ attributed to the strong $\mathrm{v}(\mathrm{C}-\mathrm{C})$ in benzene ring. Appearance of absorption band at $1310 \mathrm{~cm}^{-1}$ was assigned to amide III $\delta(\mathrm{N}-\mathrm{H})+v(\mathrm{C}=\mathrm{N})$, and $\beta(\mathrm{C}-\mathrm{H})$ peak $1221 \mathrm{~cm}^{-1}$. The region between 1103 and $916 \mathrm{~cm}^{-1}$ was the $v\left(\mathrm{CH}_{2}-\right.$ $\left.\mathrm{O}-\mathrm{CH}_{2}\right)$ of ether peak and $1018 \mathrm{~cm}^{-1}$ was the weak $\beta(\mathrm{C}-\mathrm{H})$ in benzene ring. At the same time heparin has four major absorption bands between 900 and $1700 \mathrm{~cm}^{-1}$, with one band made of two shoulders at 969 and $1017 \mathrm{~cm}^{-1}$, and three bands at 1221, 1419, and $1604 \mathrm{~cm}$ which confirms its presence in cospun PU-PLLA membranes as depicted in Figure 2B.

The FTIR results showed that no chemical reactions occurred between PLLA and PU during electrospinning as no new chemical bonds were identified

However, this FTIR analysis could not detect the presence or absence of heparin on these membranes due to overlapping peaks positions as shown in Figure 2B.

\subsection{MECHANICAL PROPERTIES}

PLLA membranes showed the highest plastic deformation compared to PU and cospun PU-PLLA membranes, while last two membranes presented a very similar elastic behavior (Figure 3A and 3B). In 
terms of tensile strength, the cospun-PU-PLLA membrane was significantly higher compared to PLLA and Z3 membranes and above the values of porcine dermis used as a reference commercial material clinically used for grafting of oral soft tissue defects (Herford, Akin, Cicciu, Maiorana, \& Boyne, 2010) ; while the last two membranes were between the range of values for porcine dermis (Figure 3C).

All membranes were tested for their ability to retain sutures and there were no significant differences between them (Figure 3D).

\subsection{CULTURE OF CELLS ON SCAFFOLDS}

Figure 4A shows the viability of cells on the uncoated membranes as assessed using Alamar Blue. It was observed that the uncoated membrane showed similar cell viability and proliferation on tissue culture plastic (TCP) (Figure 4A). There was no significant difference between the TCP grown cells and those grown on uncoated membranes during the time period studied. However, the plasma coating positively affected the growth and viability of cells seeded on the membranes and showed significantly higher cell viability and proliferation compared to TCP control (Figure 4A).

The appearance of the cells on the scaffolds is also shown In Figure 4B where cells and scaffolds are imaged by SEM and by staining with Hematoxylin and Eosin. This shows good attachment of cells to the scaffolds and H\&E shows that they have spread out on the scaffolds and appear securely attached. This was the case for both uncoated and coated scaffolds.

\subsection{EFFECT OF HEPARIN BINDING ON ANGIOGENIC ACTIVITY OF MEMBRANES}

The angiogenic potential of the membranes was assessed through use of the CAM assay. Blood vessels can be seen growing towards the plasma coated membranes (Figure 5B and D). The cell infiltration was similar for the coated and uncoated membranes and no significant difference was observed; however, the number of blood vessels for the coated membranes was significantly higher compared to the uncoated controls (Figure 5).

\subsection{EyALUATION OF MATERIALS IN ANIMALS}

Plasma coated membranes loaded with heparin and uncoated membranes were implanted subeutaneously for three time periods, 1 week, 3 weeks and 6 weeks (Figure 6A-G). It was noted that the wounds healing was completed by skin closure and there was no scarring or inflammation when observed visually (Figure 6B). There were no signs of redness and swelling after 1 week (Figure 6B and $\mathrm{C}$ ). The tissue was harvested and on opening of the subcutaneous tissue no signs of inflammation were observed at any time point (Figure 6C). After 3 weeks the wounds had healed completely and there was no scar formation with normal hair growth which covered the shaved area. To expose the implant, the hair was removed again and the skin was excised to completely remove the implanted membranes with the surrounding tissue (Figure 6D). The implants looked well embedded and could be clearly seen through the transparent subcutaneous layer (Figure 6E). At week 6 the implant site was completely indistinguishable from the rest of the body and the area was exposed by removing hair and implant was again clearly visible from the underside of the excised skin (Figure 6F and G). 


\subsubsection{HISTOLOGY OF IMPLANTS IN THE SUBCUTANEOUS TISSUE}

The implants were harvested from the rats and were fixed and processed for histology. The cross sections through the implants inside the subcutaneous tissue were stained with Hematoxylin and eosin and these confirmed that the tissue had integrated well into the skin layers (Figure 6H-P) with similar results for both coated (coated) and non-coated (not shown) membranes. It was noted that the implanted membranes were loosely embedded in the connective tissue under the skin. Six weeks after implantation.

\section{DISCUSSION}

Our aim in this study was to develop a membrane which would be useful for the surgical treatment of oronasal fistulae. To achieve this, we co-spun PU and PLLA to create mechanically flexible PU fibers interwoven with highly biocompatible PLLA. The porous nature of the membrane created by electrospinning encouraged cells to attach and proliferate and the membranes integrate well postimplantation in rat tissue. We also surface modified these electrospun membranes to enhance their ability to stimulate new blood vessels ingrowth into the membranes.

Any material designed to replace tissues for medical applications needs to have mechanical properties which can match the matrix of the host tissues without inducing any inflammation or toxicity. Also it needs to be easily processed and sterilized with an adequate shelf life (Macocinschi et al., 2011). PUs are elastomers with good biocompatibility. PU was chosen as a material that has been used extensively in medical devices and is capable of distension without distorting the surrounding tissues. Thus PUs have been used in clinical studies as implants and biomedical devices, vascular prosthesis, blood pumps, mammary prosthesis, heart valves, pace makers, intra-aortic balloons, catheters and artificial hearts (Baumgartner, Yang, \& Cooper, 1997; Hung, Wu, Chien, \& Hsu, 2009; Reddy, Kano, Maruyama, Hadano, \& Takahara, 2008; Wu et al., 2009). Many of these fistulae occur in young children who grow fast and need highly distensible materials as devices.

A combination of hydrophilic and hydrophobic properties increases the biocompatibility and hemo compatibility of PUs. Generally, solid surfaces with CAs less than $90^{\circ}$ are considered hydrophilic, while surfaces with CAs higher than $90^{\circ}$ are considered hydrophobic. The boundary between hydrophilicity and hydrophobicity for a smooth solid surface actually occurs at a CA of $65^{\circ}$ (Patel, Varilly, \& Chandler, 2010; Yoon, Flinn, \& Rabinovich, 1997). Plasma surface coated membranes has left and right contact angles of $63.1^{\circ}$ and $58.7^{\circ}$ well below $65^{\circ}$ which is an evidence of increased hydrophilic nature of the surface. Fiber diameter was significantly higher after plasma coating and proportional to the pore size reduction after plasma coating as discussed in the results section. The combination of fiber thickness and void /pore size in these membranes was optimal with around 4-5.5 um void size (Lowery, Datta, \& Rutledge, 2010) demonstrating good cell attachment and proliferation.

Additionally, all membranes were easy to handle with a suture retention strength that makes them suitable for their surgical implantation into the oral mucosal layers of the palate (Hudson \& Pickett, 
2015). The PU-PLLA membrane showed an elastic behavior which is desirable to cope with the physical distention of the oral mucosa, due to the elastomeric properties of PU. If these materials can stretch readily to accommodate the growth of the child, then they will be less likely to hinder or restrain facial development which is known to occur with bone implants which become fused in place leading to distortion of the growing face (Guerrero, 2012). The combination of the two polymers increased the tensile strength of the cospun membrane above the values of a commercial porcine tissue. In addition, the most relevant mechanical properties will need to be studied in vivo, which will be determined by the degradation rate of PLLA versus the production of new matrix. Therefore, due to an initial hydrolysis of PLLA after implantation, a higher tensile strength may be desirable to maintain the loadbearing properties while cells infiltrate, and new matrix is produced.

The fabricated membranes were modified by surface plasma polymerization showing heparin binding and promoting vascularization. The main problem in many implanted membranes is a lack of vascularization. Heparin and VEGF are some of many compounds used to enhance neo vascularization in membranes. Natural and synthetic heparin are more translatable compared to the much more expansive and less available recombinant growth factors. One of the many current techniques for surface functionalization is a layer by layer approach (Gigliobianco et al., 2015). In this technique alternative layers of PEI and heparin are built (Easton, Bullock, Gigliobianco, McArthur, \& MacNeil, 2014) and then we used the chick CAM to demonstrate that the coated materials were angiogenic.

We confirmed through histology that more extensive infiltration of chick cells took place into the plasma coated membrane compared to the uncoated PU-PLLA membrane. In our studies plasma coated PU-PLLA membranes showed twice as much neovascularization as uncoated membrane within 7 days after implantation into the CAM assay.

In addition, these fibrous polymer membranes were highly biocompatible promoting cell attachment and proliferation, and we further confirmed cell infiltration into the membranes through hematoxylin and eosin staining. In vivo implantation of these membranes in rat sub-cutaneous tissue also demonstrated good integration into the body. The animals did not show any signs of discomfort or any behavioral abnormality throughout the period of observation. Wounds healed with completely closure with no scar formation and a normal growth of hair by week 3. Histological sections of implants with surrounding tissue showed results consistent with the visual observation of the implant area in rats. The sections showed growing hair follicles with normal epidermis, dermis and implanted membrane infiltrated with cells. The dermis also showed dermal appendages and sebaceous glands as for the control sham operated skin tissue.

A limitation of this study was the animal model selected, since the rats selected present a good wound healing (native tissue repair) and no differences were observed in that process when the coated or uncoated composite membranes were implanted. This model demonstrated initial safety and biocompatibility of the membranes produced. Future studies should ideally involve more functional animal models- such as diabetic animals where wound healing is slow, and in particular implantation into a physiologically 
relevant animal model of cleft palate such as the non-syndromic cleft palate mouse (Wnt9b-/clf1) (Juriloff \& Harris, 2008) .

\section{Conclusions}

In conclusion, the production of a composite electrospun porous membrane which is surface modified results in a distensible material which can promote angiogenesis and is well tolerated when implanted in animals. We conclude that this material should be considered for further development as a promising approach for the surgical treatment of oral nasal fistulas.

\section{Acknowledgements}

The authors would like to express their gratitude to the PAK-UK fellowship program from COMSATS Institute of Information technology, Lahore and support of HEC Pakistan. We would also like to thank Waleed Mustafa for his help during in vivo experiments and Faisal Manzoor for SEM imaging.

\section{CONFLiCT OF INTEREST STATEMENT}

The authors of this manuscript do not have any conflicts of interest.

Author contribution statement

1. Faiza Sharif

Corresponding author, conceived the research idea and executed all steps till completion and wrote the manuscript

2. Sabiniano Roman

Helped in electrospinning and mechanical strength test of membranes, contributed heavily in write up

3. Anila Asif

Contributed through purchase of polymers and FTIR analyses

4. Giulia Gigliobianco

Contributed through plasma polymerization of membranes

5. Sarah Ghafoor

Helped in in vivo surgical procedures and maintaining the animal model before and after surgical procedures in her facility 


\section{Muhammad Tariq}

Provided his cell culture facility with all the provisions and supplies to carry out cytotoxicity and biocompatibility evaluation. Without his support cell studies were not possible

7. Saadat Anwer Siddiqui

Supported through SEM analysis of samples before and after cell cultures when the SEM was not available in our lab

8. Farrukh Mahmood

Clinical expert who guided us through the clinical requirements of regenerative materials. His experience with recurrent fistulation and the feedback of expansive acellular dermis not available in Pakistan for most patients lead us to this research work.

9. Ihtesham Ur Rehman

Our mentor in biomaterial research in Pakistan who established this facility and guided us through every step

10. Sheila MacNeil

My supervisor and mentor in biomaterial research who guided us through every step of material development and contributed heavily in write up 


\section{REFERENCES}

Alperin, C., Zandstra, P. W., \& Woodhouse, K. A. (2005). Polyurethane films seeded with embryonic stem cell-derived cardiomyocytes for use in cardiac tissue engineering applications. Biomaterials, 26, 7377-7386. doi: 10.1016/j.biomaterials.2005.05.064

Badami, A. S., Kreke, M. R., Thompson, M. S., Riffle, J. S., \& Goldstein, A. S. (2006). Effect of fiber diameter on spreading, proliferation, and differentiation of osteoblastic cells on electrospun poly(lactic acid) substrates. Biomaterials, 27(4), 596-606. doi: S0142-9612(05)00524-7 [pii];10.1016/j.biomaterials.2005.05.084 [doi]

Baumgartner, J. N., Yang, C. Z., \& Cooper, S. L. (1997). Physical property analysis and bacterial adhesion on a series of phosphonated polyurethanes. Biomaterials, 18(12), 831-837. doi: S0142961296001974 [pii]

Bykowski, M. R., Naran, S., Winger, D. G., \& Losee, J. E. (2015). The Rate of Oronasal Fistula Following Primary Cleft Palate Surgery: A Meta-Analysis. Cleft Palate Craniofac.J., 52(4), e81-e87. doi: 10.1597/14-127 [doi]

Chuenjitkuntaworn, B., Inrung, W., Damrongsri, D., Mekaapiruk, K., Supaphol, P., \& Pavasant, P. (2010). Polycaprolactone/hydroxyapatite composite scaffolds: preparation, characterization, and in vitro and in vivo biological responses of human primary bone cells. J.Biomed.Mater.Res.A, 94(1), 241-251. doi: 10.1002/jbm.a.32657 [doi]

Coombes, A. G. A., \& Meikle, M. C. (1994). Resorbable synthetic polymers s replacements for bone graft. Clinical Materials, 17(1), 35-67.

de Castro, C. H., de Souza, L. N., \& Fernandes Santos, M. M. (2015). Use of the buccal fat pad as free graft for closure of oronasal fistula in a cleft palate patient. J.Craniofac.Surg., 26(1), e14-e16. doi: 10.1097/SCS.0000000000001225 [doi]

Easton, C. D., Bullock, A. J., Gigliobianco, G., McArthur, S. L., \& MacNeil, S. (2014). Application of layer-by-layer coatings to tissue scaffolds - development of an angiogenic biomaterial. Journal of Materials Chemistry B, 2(34), 5558-5568. doi: 10.1039/C4TB00448E

Funayama, E., Yamamoto, Y., Nishizawa, N., Mikoya, T., Okamoto, T., Imai, S., . . Oyama, A. (2014). Important points for primary cleft palate repair for speech derived from speech outcome after three different types of palatoplasty. Int.J.Pediatr.Otorhinolaryngol., 78(12), 2127-2131. doi: S0165-5876(14)00526-6 [pii];10.1016/j.ijporl.2014.09.021 [doi]

Gigliobianco, G., Chong, C. K., \& MacNeil, S. (2015). Simple surface coating of electrospun poly-Llactic acid scaffolds to induce angiogenesis. J.Biomater.Appl., 30(1), 50-60. doi: 0885328215569891 [pii]; $10.1177 / 0885328215569891$ [doi]

Guerrero, C. A. (2012). Cleft lip and palate surgery: 30 years follow-up. Annals of maxillofacial surgery, 2(2), 153-157. doi: 10.4103/2231-0746.101342

Hardwicke, J. T., Landini, G., \& Richard, B. M. (2014). Fistula incidence after primary cleft palate repair: a systematic review of the literature. Plast.Reconstr.Surg., 134(4), 618e-627e. doi: 10.1097/PRS.0000000000000548 [doi];00006534-201410000-00034 [pii]

Herford, A. S., Akin, L., Cicciu, M., Maiorana, C., \& Boyne, P. J. (2010). Use of a porcine collagen matrix as an alternative to autogenous tissue for grafting oral soft tissue defects. J Oral Maxillofac Surg, 68(7), 1463-1470. doi: 10.1016/j.joms.2010.02.054

Hosseinabad, H. H., Derakhshandeh, F., Mostaajeran, F., Abdali, H., Davari, H. A., Hassanzadeh, A., \& Kummer, A. W. (2015). Incidence of velopharyngeal insufficiency and oronasal fistulae after cleft palate repair: A retrospective study of children referred to Isfahan Cleft Care Team between 2005 and 2009. Int.J.Pediatr.Otorhinolaryngol., 79(10), 1722-1726. doi: S01655876(15)00370-5 [pii];10.1016/j.ijporl.2015.07.035 [doi]

Hudson, J. W., \& Pickett, D. O. (2015). A 5-Year Retrospective Review of Primary Palatoplasty Cases Utilizing an Acellular Collagen Interpositional Graft. Journal of Oral and Maxillofacial Surgery, 73(7), 1393-1393.

Hung, H. S., Wu, C. C., Chien, S., \& Hsu, S. H. (2009). The behavior of endothelial cells on polyurethane nanocomposites and the associated signaling pathways. Biomaterials, 30(8), 1502-1511. doi: S0142-9612(08)00930-7 [pii];10.1016/j.biomaterials.2008.12.003 [doi] 
Juriloff, D. M., \& Harris, M. J. (2008). Mouse genetic models of cleft lip with or without cleft palate. Birth Defects Research Part A: Clinical and Molecular Teratology, 82(2), 63-77. doi: doi:10.1002/bdra.20430

Kahraman, A., Yuce, S., Kocak, O. F., Canbaz, Y., Guner, S. I., Atik, B., \& Isik, D. (2014). Comparison of the fistula risk associated with rotation palatoplasty and conventional palatoplasty for cleft palate repair. J.Craniofac.Surg., 25(5), 1728-1733. doi: 10.1097/SCS.0000000000000967 [doi]

Lowery, J. L., Datta, N., \& Rutledge, G. C. (2010). Effect of fiber diameter, pore size and seeding method on growth of human dermal fibroblasts in electrospun poly( $\varepsilon$-caprolactone) fibrous mats. Biomaterials, 31(3), 491-504. doi: https://doi.org/10.1016/j.biomaterials.2009.09.072

Macocinschi, D., Filip, D., \& Vlad, S. (2011). Natural-Based Polyurethane Biomaterials for Medical Applications: INTECH Open Access Publisher.

Mossey, P. (2004). Addressing the global challenges of craniofacial anomalies. report of a WHO

meeting on international collaborative research on craniofacial anomalies. Technical Report, Avenue Appia, 1211.

Ott, H. C., Matthiesen, T. S., Goh, S.-K., Black, L. D., Kren, S. M., Netoff, T. I., \& Taylor, D. A. (2008). Perfusion-decellularized matrix: using nature's platform to engineer a bioartificial heart. Nat Med, 14(2), 213-221. doi: http://www.nature.com/nm/journal/v14/n2/suppinfo/nm1684 S1.html

Patel, A. J., Varilly, P., \& Chandler, D. (2010). Fluctuations of water near extended hydrophobic and hydrophilic surfaces. The journal of physical chemistry. B, 114(4), 1632-1637. doi: 10.1021/jp909048f

Porter, J. R., Ruckh, T. T., \& Popat, K. C. (2009). Bone tissue engineering: a review in bone biomimetics and drug delivery strategies. Biotechnol.Prog., 25(6), 1539-1560. doi: $10.1002 / \mathrm{btpr} .246$ [doi]

Reddy, T. T., Kano, A., Maruyama, A., Hadano, M., \& Takahara, A. (2008). Thermosensitive transparent semi-interpenetrating polymer networks for wound dressing and cell adhesion control. Biomacromolecules., 9(4), 1313-1321. doi: 10.1021/bm701390f [doi]

Sadhu, P. (2009). Oronasal fistula in cleft palate surgery. Indian J Plast Surg, 42(Suppl), S123-S128.

Sombatmankhong, K., Sanchavanakit, N., Pavasant, P., \& Supaphol, P. (2007). Bone scaffolds from electrospun fiber mats of poly(3-hydroxybutyrate), poly(3-hydroxybutyrate-co-3hydroxyvalerate) and their blend. Polymer, 48(5), 1419-1427.

Wang, S., Cao, X., Shen, M., Guo, R., Banyai, I., \& Shi, X. (2012). Fabrication and morphology control of electrospun poly(gamma-glutamic acid) nanofibers for biomedical applications. Colloids Surf.B Biointerfaces., 89, 254-264. doi: S0927-7765(11)00552-2

[pii];10.1016/j.colsurfb.2011.09.029 [doi]

Wu, Z., Chen, H., Huang, H., Zhao, T., Liu, X., Li, D., \& Yu, Q. (2009). A facile approach to modify polyurethane surfaces for biomaterial applications. Macromol.Biosci., 9(12), 1165-1168. doi: 10.1002/mabi.200900221 [doi]

Yoon, R.-H., Flinn, D. H., \& Rabinovich, Y. I. (1997). Hydrophobic Interactions between Dissimilar Surfaces. Journal of Colloid and Interface Science, 185(2), 363-370. doi: https://doi.org/10.1006/jcis.1996.4583

Yoshimoto, H., Shin, Y. M., Terai, H., \& Vacanti, J. P. (2003). A biodegradable nanofiber scaffold by electrospinning and its potential for bone tissue engineering. Biomaterials, 24(12), 20772082. doi: S014296120200635X [pii]

Zdrahala, R. J., \& Zdrahala, I. J. (1999). Biomedical applications of polyurethanes: a review of past promises, present realities, and a vibrant future. J Biomater Appl, 14, 67-90.

Zhang, D., Giese, M. L., Prukop, S. L., \& Grunlan, M. A. (2011). PCL-based Shape Memory Polymers with Variable PDMS Soft Segment Lengths. Journal of polymer science.Part A, Polymer chemistry, 49(3), 754-761. 



Figure 1A. Top: Scanning Electron Micrographs of membranes. A. Uncoated PU- PLLA cospun membrane. B Plasma coated PU- PLLA cospun membrane. Bottom: Fiber thickness analysis of PU plain membrane, PLLA plain membrane, uncoated and plasma coated cospun membranes assessed by image J software. Oneway ANOVA multiple comparisons test (Sidak's multiple comparisons test) (*** statistically significant difference between each group, $\mathrm{P}$ value 0.0001 ). Figure $1 \mathrm{~B}$

Hydrophobicity and hydrophilicity of (a) PU PLA Uncoated and (b) Coated membranes measured

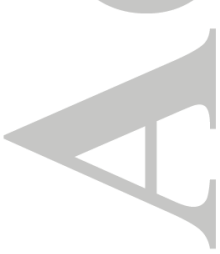
through contact angle analysis. 

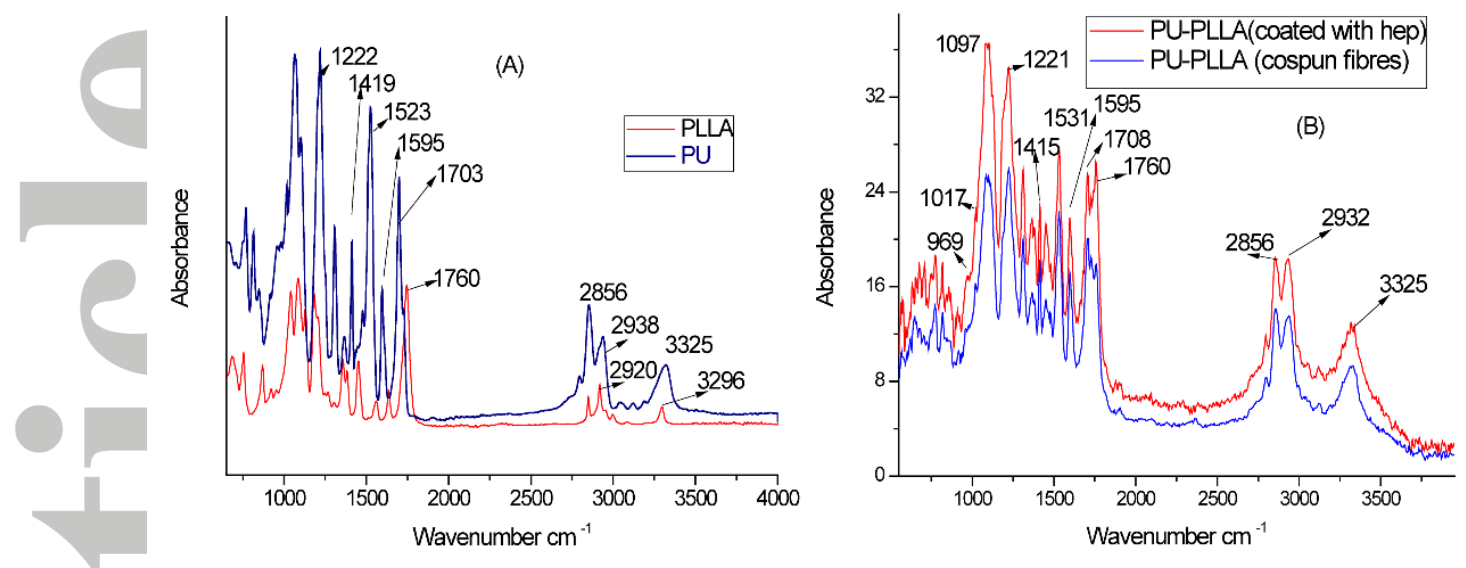

Figure 2. (A) Combined FTIR spectra of PLLA, and PU on common scale, (B) Combined FTIR of PU-PLLA cospun membranes and PU-PLLA coated with heparin. 



Figure 3. Mechanical properties of PU-PLLA cospun membranes in comparison with plain PLLA and PU electrospun membranes. (A) Stress-strain curves of the cyclic test. (B) Plastic deformation. (C) Ultimate tensile strength in $\mathrm{N} / \mathrm{mm}^{2}\left({ }^{*} \mathrm{p}<0.05\right)$. (D) Suture retention test. 
Cell viability on PU-PLA membranes

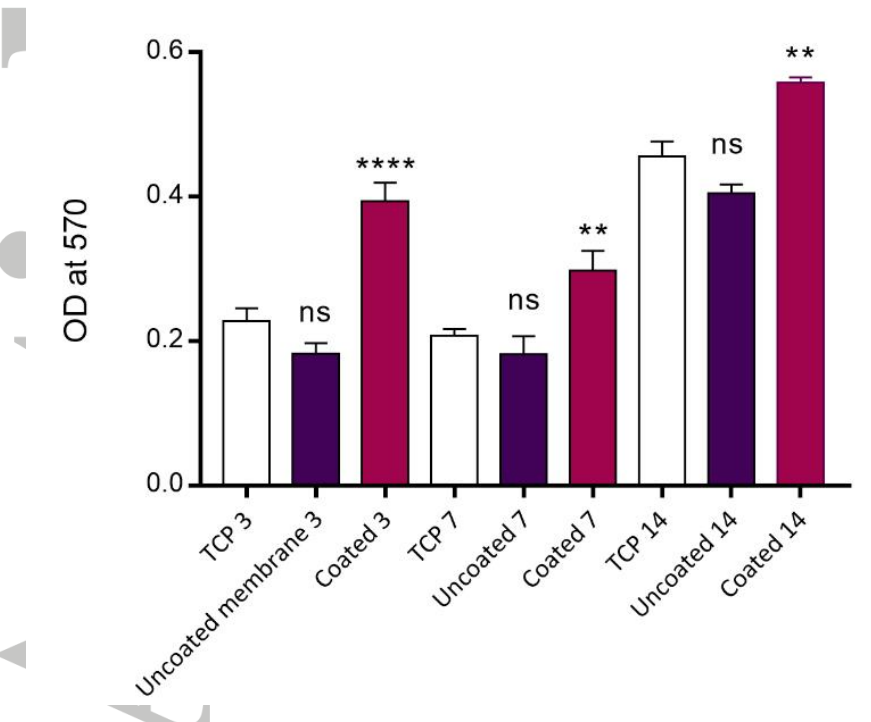



Figure 4a. MC 3T3 cell viability assay on coated and non-coated membranes. Day 3, 7 and 14 (black bars) compared to controls TCP (white bars). Error bars represent standard error of the mean $(n=3$, $* \mathrm{p}<0.01)$ ns: not significant.

Figure 4b. Top: Scanning electron micrographs of MC3T3 cells cultured on electrospun PU-PLLA membranes for 3 days. (A) Cells on coated PU-PLLA membrane. (B) Cells on uncoated fibrous electrospun membrane Bottom: Whole mount H\&E images of PU-PLLA membranes 3 days after cell culture with MC3T3 cells. (C) Plasma coated membranes with nuclei stained in blue. (D) Uncoated cospun membranes with cells. Scale bar $=100 \mu \mathrm{m}$ from Figure A to F. 

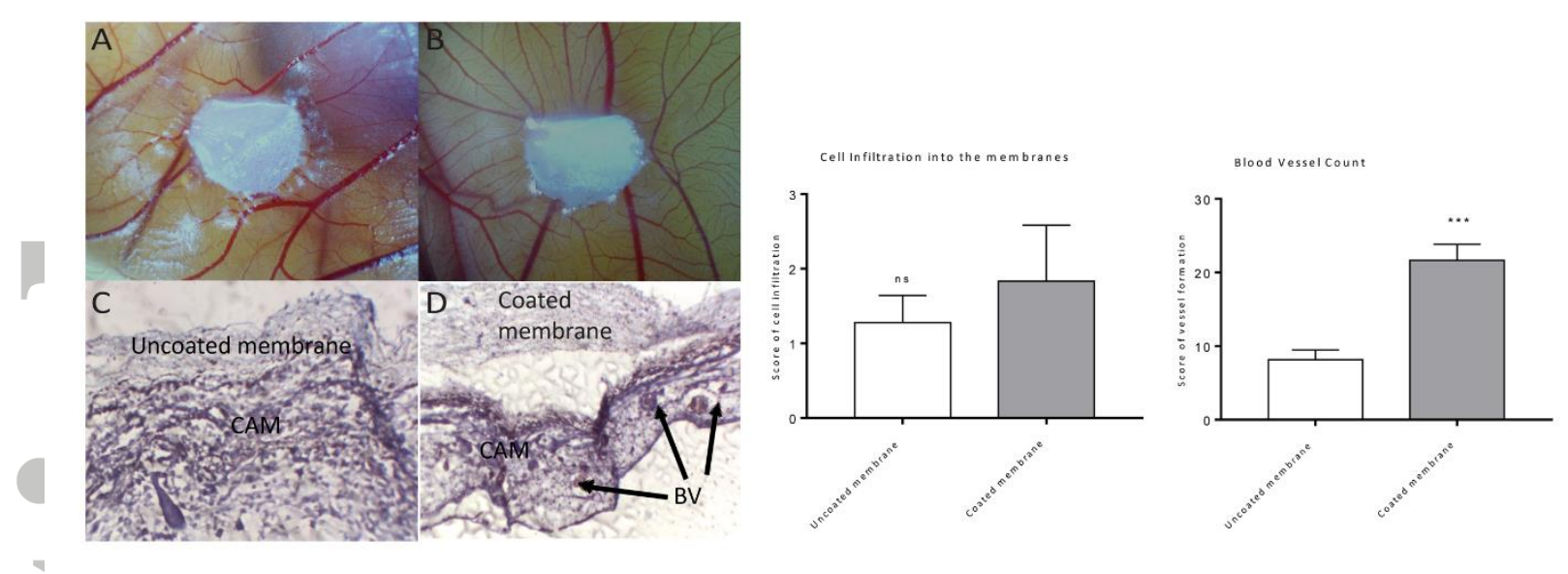

Figure 5a. Chorio allantoic membrane assay (CAM) for vascularization, in chick embryo model. (A) Uncoated PU-PLLA membrane showing normal vascularization with no blood vessel growth towards the membrane, (B) Plasma coated PU_PLLA membrane after 7 days of implantation, blood vessels converging towards the membrane indicating neovascularization. (C) Histology of uncoated membrane after 7 days of implantation. (D) Plasma coated membrane with neovascularization (arrows showing small blood vessels BV).

Figure 5b. Bar graph showing statistical analysis of cell infiltration scoring between categories 1-3 in uncoated and coated membranes (left), and total number of blood vessels on coated and uncoated membranes (right); ns: not significant, $* * * \mathrm{P}$ value $<0.0001$.

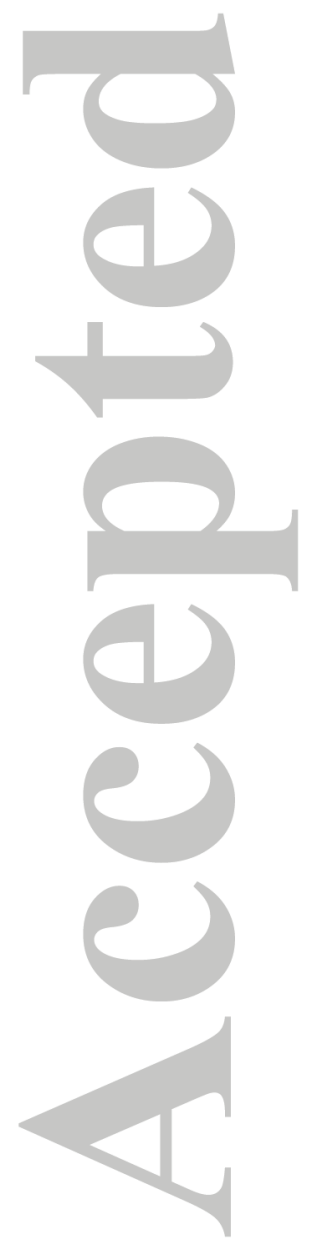



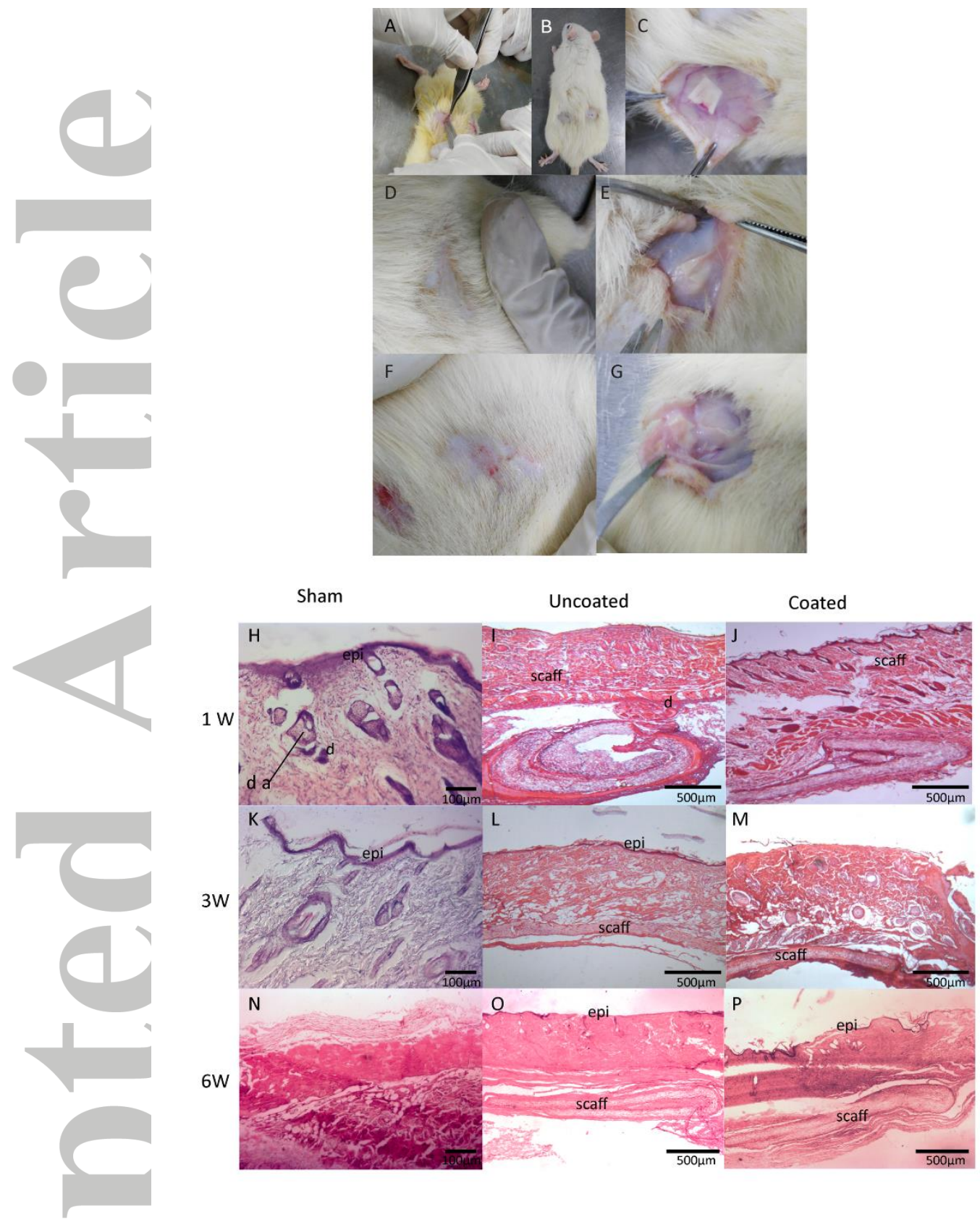

Figure 6a. Method of in vivo implantation of plasma coated and uncoated membranes in rats. Figure 6A-G whole mount images of surgical site. (A) Implantation site. (B) Healed wound after 1 week with no hair regrowth. (C) Harvesting of implant with surrounding skin at 1 week. (D) Implant site at 3 weeks with hair growth. (E) Implant embedded in the subcutaneous tissue after 3 weeks. (F) Implant site after 6 weeks of surgical implantation, with hair shaved off. (G) Harvesting of implant from the skin after 6 weeks.

Figure 6b. Histology of implanted membrane at different time points (H\&E staining), all coated membranes were loaded with heparin pre-implantation. (H-J) Histology of skin after 1 week. $(\mathrm{H})$ Sham operated (I) Morphology of skin after 1 week of implantation of uncoated membrane. (J) Skin implanted with plasma coated membrane. (K-M) 3 weeks after implantation. (K) Sham operated. (L) Skin with uncoated membrane. (M) Coated membrane implanted for 3 weeks. (N-P) 6 weeks after implantation of membrane. (N) Sham operated. (O) Uncoated Membrane implant. (P) Coated membrane after 6 weeks of implantation. Epi (epidermis), d (dermis), a (appendages), scaff, (Scaffold/membrane). Scale bar $100 \mu \mathrm{m}$ and $500 \mu \mathrm{m}$. 